\title{
COMUNIDADES DE MACROINVERTEBRADOS ACUÁTICOS EN QUEBRADAS DE LA ISLA DE PROVIDENCIA, MAR CARIBE COLOMBIANO
}

\author{
AQUATIC MACROINVERTEBRATES COMMUNITIES OF THE STREAMS OF \\ PROVIDENCE ISLAND, CARIBBEAN SEA OF COLOMBIA
}

Daniela Cortés-Guzmán y Rodulfo Ospina-Torres

\begin{abstract}
RESUMEN
A pesar del uso extendido de las comunidades de macroinvertebrados dulceacuícolas en estudios básicos y aplicados de ecología acuática, es poco lo que se conoce para la Isla de Providencia, más aun cuando la biota de las islas es de interés por sus posibles relaciones con el continente y con islas cercanas. El presente estudio caracterizó la composición y la estructura de las comunidades de macroinvertebrados dulceacuícolas de la Isla durante tres periodos de muestreo relacionados con el ciclo hidrológico e identificó las distribuciones geográficas de los elementos faunísticos encontrados. Se seleccionaron cinco sitios (Lazy Hill, Bailey, Fresh Water, El Pico y Old Town) en los cuales se midieron variables físicas y químicas y se recolectaron individuos en cada microhábitat disponible. Posteriormente se calcularon descriptores de la comunidad (riqueza específica y abundancia) e índices de diversidad (diversidad de Margalef, dominancia de Simpson y equidad de Shannon-Weiner) y se aplicaron pruebas de Kruskal Wallis para establecer las diferencias en las abundancias entre las épocas de muestreo y entre los sitios. Un ACC se realizó para establecer la asociación de los organismos con las variables físicas y químicas. Se encontró que los sitios de Lazy Hill y El Pico son los que representan mayor diversidad (DMg: 7.15 y 8.40, respectivamente) y pueden ser un refugio importante para las comunidades en la época de sequía. Las distribuciones geográficas no permitieron reconocer endemismos a nivel genérico, sino que las comunidades estudiadas son una combinación de elementos cosmopolitas centro y suramericanos principalmente. La estacionalidad tiene un efecto notable sobre la composición de las comunidades, siendo mayor en los cuerpos de agua con menos obras de intervención como Lazy Hill y El Pico.
\end{abstract}

PALABRAS CLAVE: Caribe colombiano, Isla de Providencia, macroinvertebrados dulceacuícolas

\begin{abstract}
Despite the widespread use of aquatic macroinvertebrates in basic and applied studies of aquatic ecology, little is known of Providence, even more when the biota of the islands represents itself a subject of interest because of its relationships with the mainland and other nearby islands. This study described the composition and structure of the island's aquatic macroinvertebrates communities during three periods related to hydrologic cycle, and identified the geographic distributions of the faunal elements found. Five sites of the island (Lazy Hill, Bailey, Fresh Water, El Pico and Old Town) in which physical and chemical parameters were measured, and organisms were collected in the available microhabitat. Descriptors (species richness and abundance) and diversity indexes (Margalef diversity index, Simpson's dominance index and Shannon-Wiener index) of the population were calculated. Kruskal Wallis test was applied to establish differences between abundance and seasons, and a CCA test for establish the association of the organisms with physical and chemical parameters. It was found that Lazy Hill and The Peak are the most diverse sites (DMg 7.15 and 8.40, respectively) and they may be a valuable refuge for the communities during the dry season. Geographic distributions did not allow recognition of endemism at the genera level, but the communities studied are mainly a combination of cosmopolitan and Central and South American elements. Seasonality has a remarkable effect on the composition of communities, being greater at sites with less intervention, as Lazy Hill and The Peak.
\end{abstract}

KEY WORDS: Colombian Caribbean, Providence Island, aquatic macroinvertebrates 


\section{INTRODUCCIÓN}

Los sistemas lóticos brindan importantes servicios ecosistémicos, entre ellos el de hábitat, ya que sustentan una gran diversidad biótica, incluida la de macroinvertebrados bentónicos. Desde un punto de vista funcional, los macroinvertebrados pueden jugar un papel preponderante en la red de interacciones de estos ecosistemas y en el flujo de recursos alimenticios (Domínguez y Fernández, 2009). Este hecho hace que las comunidades de macroinvertebrados puedan reflejar ciertos cambios en el entorno, de manera que éstas han sido ampliamente utilizadas en estudios de ecología básica y aplicada (Riss et al., 2002; Muñoz-Riveaux et al., 2003; Naranjo-López et al., 2005; Pérez-Pompa et al., 2012).

En el caso particular de las quebradas temporales, los organismos que las habitan tienen estrategias especiales de supervivencia debido a las condiciones de estrés hídrico que deben soportar. Además, representan sistemas de gran diversidad, pues son ambientes extremos que aceleran las tasas de diversificación, sumado a que históricamente han podido actuar como refugios y rutas de dispersión (Dudley-Williams, 2006).

Los estudios sobre las comunidades de macroinvertebrados bentónicos dulceacuícolas para la Isla de Providencia son escasos, aunque existen algunos registros, entre ellos de crustáceos del género Macrobrachium (Valencia y Campos, 2007). Para otras islas del Caribe como Puerto Rico se estudió la distribución espacial de crustáceos y moluscos (Pyron et al., 1999; Fièvet et al., 2001; Bass, 2003; Blanco y Scatena, 2006) y en Cuba, en bioindicación de macroinvertebrados bentónicos utilizando el índice BMWP-Cub (Muñoz-Riveaux et al., 2003; Naranjo-López et al., 2005; Pérez-Pompa et al., 2012). Los resultados se constituyen como una importante herramienta que ha contribuido al conocimiento del estado de conservación de los ríos y una evaluación de la biodiversidad que alberga estos ecosistemas y que no han sido tenidos en cuenta. Por lo tanto, el presente trabajo tuvo como objetivo caracterizar la composición y definir la estructura de las comunidades de macroinvertebrados de cinco cuerpos de agua dulce de la Isla de Providencia y establecer sus diferencias en tres periodos hidrológicos. Se estudió la composición y estructura de la comunidad mediante índices ecológicos y descriptores. Así mismo se establecieron las preferencias de microhábitat y la distribución geográfica de los taxones encontrados.
La estacionalidad de las lluvias en la isla y el carácter intermitente de sus cuerpos de agua, permiten plantear la hipótesis de que las comunidades de macroinvertebrados en las épocas de lluvias aumentaran su abundancia y su diversidad. Sería de esperar que en la época de lluvias hagan su aparición taxones de aguas altas y que se encuentren diferentes taxones dominantes en cada época.

\section{MATERIALES Y MÉTODOS}

\section{Área de estudio}

El Archipiélago de San Andrés y Providencia, Colombia, está ubicado en el mar Caribe Centro-Occidental y tiene menos del $1 \%$ de su territorio como áreas emergidas. Es un conjunto de islas de origen volcánico, con un régimen climático monomodal que es afectado por huracanes y vientos del este del Caribe y por la zona de convergencia intertropical (ZCIT). El periodo lluvioso está comprendido entre mayo y diciembre, mientras que el periodo seco comprende de enero hasta abril (CORALINA-INVEMAR, 2012). La Isla de Providencia, en particular, se caracteriza por presentar una morfología de colinas y pequeñas montañas. Providencia es la única isla del Archipiélago que tiene pequeñas quebradas, las cuales aumentan sus caudales en la época lluviosa que mantienen manantiales durante todo el año (CORALINA-INVEMAR, 2012), y se constituyen como una de las fuentes de agua dulce más importantes para los habitantes de la Isla y para la biota en general. El carácter intermitente de las quebradas, hace que durante los periodos de bajas lluvias, los caudales disminuyan drásticamente, y sólo permanezcan pequeños pozos aislados. Adicionalmente, las obras de intervención del caudal en algunas quebradas, impiden el flujo normal del agua, generando pozos.

Se realizaron tres muestreos en cinco sitios de la Isla: Lazy Hill, Bailey, Fresh Water, El Pico y Old Town. Se obtuvieron los datos de precipitación del 2013 de la estación meteorológica de San Felipe del IDEAM (Figura 1). Allí se observó que de enero a mayo se presentó muy baja precipitación, correspondiente al periodo de sequía o lluvias bajas. En junio se presentaron algunas precipitaciones, que volvieron a disminuir drásticamente en julio, cuando se realizó el primer muestreo. Septiembre y octubre, cuando ocurrió el máximo pico de precipitación, se realizó el segundo muestreo. El tercer muestreo se realizó en enero de 2014, correspondiente a la finalización de las lluvias, o inicio de la sequía. 


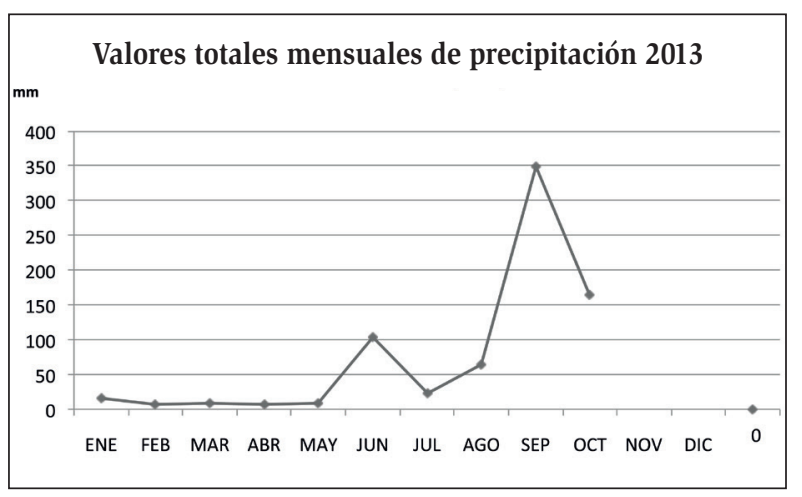

Figura 1. Valores totales mensuales de precipitación 2013. Estación San Felipe, Providencia. IDEAM- Instituto de hidrología, meteorología y estudios ambientales.

\section{Descripción de los sitios}

1. Lazy Hill es un sitio conformado por una quebrada intermitente y un manantial perenne (fuente de agua natural que brota de entre las rocas). La vegetación circundante sombrea aproximadamente el $90 \%$ del canal y está compuesta mayoritariamente por árboles y arbustos con elementos nativos como especies del género Acacia (Fabaceae), así como espacios intervenidos con pequeños cultivos de pancoger pertenecientes a las fincas aledañas. En el primer muestreo el sistema estaba compuesto por pozos aislados, que recuperaron su conectividad con el flujo de agua generado por las lluvias del segundo periodo, y se mantuvieron así hasta el tercer periodo. El caudal durante el segundo periodo fue de $0.012 \mathrm{~m}^{3} / \mathrm{s}$, pero se redujo notablemente para el tercer periodo $\left(1.24 \times 10^{-3} \mathrm{~m}^{3} / \mathrm{s}\right)$.

2. La quebrada de Bailey se encuentra en un sector con abundante vegetación nativa, predominantemente arbórea, que incluye Acacia (Fabaceae), indio en cueros Bursera sumaruba (Burseraceae) y algunas especies del género Hibiscus (Malvaceae). No se ve afectada por la época hidrológica debido a que está aislada del cauce de una quebrada por las estructuras de captación que la rodean y cubierta por una malla de angeo, por lo que se mantuvo igual en todos los muestreos en sus condiciones físicas y no parece estar sujeta a los cambios temporales debidos al régimen de pluviosidad.

3. La quebrada Fresh Water tiene el caudal controlado por la represa ubicada aguas arriba, y muestra una fuerte intervención, de tal manera que no se muestran cambios físicos evidentes entre las épocas hidrológicas. Algunos elementos de la vegetación son nativos, tales como especies de Acacia (Fabaceae) y ceiba de leche Hura crepitans (Euphorbiaceae), con abundantes bejucos y alta abundancia de árboles y frutos de mango Mangifera indica (Anacardiaceae).

4. En la quebrada El Pico la vegetación es en su mayoría nativa, predominantemente arbórea, con presencia de Acacia (Fabaceae), Hibiscus (Malvaceae), Ficus (Moraceae), Bursera simaruba (Burseraceae) y alta abundancia de mango Mangifera indica (Anacardiaceae). Sin embargo, en la parte baja de la cuenca el paisaje está fuertemente intervenido, con pastizales, cultivos de pancoger y ganadería. No se observaron cambios físicos evidentes en la quebrada entre los periodos de muestreo, probablemente debido a los tubos de captación de agua instalados por el municipio entre el primer y el segundo muestreo, que no permitieron que aumentara el caudal con las lluvias.

5. En la quebrada de Old Town hay dos cisternas dispuestas para el abastecimiento de agua del sector que interrumpen el flujo de la quebrada, por lo tanto no se mostraron cambios físicos entre los muestreos los cuales se realizaron aguas arriba de dichas cisternas. La vegetación es arbórea en su mayoría, con presencia de Hibiscus (Malvaceae), Acacia (Fabaceae), Mangifera indica en abundancia (Anacardiaceae) y palmas del género Acoelorraphe c.f. (Arecaceae).

\section{Muestreos}

En cada sitio se identificaron los microhábitats, que se clasificaron de acuerdo al sustrato predominante (80\% o más de cobertura) en: raíces, cantos rodados (menores a $10 \mathrm{~cm}$ de diámetro), hojarasca, musgos, rocas (mayores a $10 \mathrm{~cm}$ de diámetro), limo y superficie del agua (pleuston). Se midieron las variables físicas y químicas: oxígeno disuelto (mg/L) (prueba de Oxígeno Aquamerck Método volumétrico según Winkler), alcalinidad (mmol/L) (prueba de alcalinidad Aquamerck - Método volumétrico), conductividad $(\mu \mathrm{S} / \mathrm{cm})$, temperatura $\left({ }^{\circ} \mathrm{C}\right)$ (sonda WTW Cond 315i) y pH (sonda Hanna HI 8314). Los organismos se recolectaron utilizando una red Surber de 144 $\mathrm{cm}^{2}$ con poro de malla de $250 \mu \mathrm{m}$, con tres réplicas en cada microhábitat. Los macroinvertebrados se preservaron en alcohol etílico (96\%) y posteriormente se determinaron al nivel más detallado posible, usando las claves de Wiederholm $(1983,1986)$, Epler (1992), Ruiz-Moreno et al. (2000), Heckman (2001, 2006), Triplehorn y Johnson (2005), Borkent y Spinelli (2007) y Domínguez y Fernández (2009) y se consultaron especialistas en moluscos y ácaros. 


\section{Tratamiento de datos}

Se calcularon los descriptores: riqueza específica y abundancia absoluta y relativa, y los índices: diversidad de Margalef DMg, dominancia de Simpson (Cinv) y equidad de Shannon-Wiener H' (ln) para cada sitio en cada época y para cada sitio con la sumatoria de los tres muestreos. Las abundancias absolutas y relativas de cada especie o morfoespecie en cada sitio de muestreo y para cada época pueden ser consultadas en la página web https:// sites.google.com/site/macroinvertebradosunal/home). Se realizaron pruebas de Kruskal Wallis para comprobar diferencias en las abundancias entre los periodos en cada uno de los sitios y se aplicó la misma prueba para comprobar diferencias entre los cinco sitios. Se analizaron las diferencias en la composición de la comunidad en las tres épocas y las preferencias de microhábitat. Para cada sitio de muestreo se analizaron las variables físicas y químicas y los cambios de estas entre las épocas y se realizó un Análisis de Correspondencia Canónica ACC (número de permutaciones: 999) para conocer las relaciones entre estas variables y las abundancias de organismos. Todos los análisis se realizaron en el programa PAST ${ }^{\circledR}$ versión 3.0.

Utilizando información bibliográfica (Woolley, 1969; Ashe et al., 1987; Harris, 1990; DeJong et al., 2001; García, 2002; Taylor, 2003; Smit, 2004; Hájek y Ŝt’astnŷ, 2005; Sganga y Angrisano, 2005; Heckman, 2006; Michat y Torres, 2006; Bartsch, 2008; Oyewo y Sæther, 2008; Pérez et al., 2008; Alarie et al., 2009; Cartwright, 2009; Domínguez y Fernández, 2009; Nessimian y Dumas, 2010; Toledo et al., 2011; Palacino-Rodríguez, 2011; Blahnik y Holzenthal, 2012; Deler-Hernández et al., 2013) se identificaron las distribuciones geográficas y las posibles regiones de origen de los taxones identificados a nivel de género. Estos se clasificaron en una de las siguientes categorías: distribución norteamericana, distribución centroamericana, distribución suramericana o cosmopolita.

\section{RESULTADOS}

Se recolectó un total de 11637 individuos distribuidos en 122 especies y morfoespecies, 109 géneros y 79 familias. Siete de estas especies o morfoespecies fueron comunes a los cinco sitios de muestreo (Chironomus sp., Dicrotendipes sp., Fittkauimyia sp., Nilotanypus sp., Microvelia sp., Oligochaeta morfoespecie 1, Pyrgophorus platyrachis). Los grupos más sobresalientes en cuanto a riqueza fueron Díptera (24\%), Acari (24\%), Coleóptera (23\%) y Trichoptera (11\%) (Figura 2a) y los más sobresalientes en cuanto a abundancia relativa fueron Gastropoda (41\%), Díptera (23\%) y Hemíptera (16\%) (Figura 2b). La prueba de Kruskal Wallis no mostró diferencias significativas entre las abundancias de los cinco sitios ( $\mathrm{p}>0.05 ; \mathrm{n}=122, \mathrm{~K}-\mathrm{W}=4.403)$. La mayor diversidad se encontró en Lazy Hill (DMg: 7.15) y en El Pico (DMg: 8.40), mientras que los menores se presentaron en Fresh Water (DMg: 2.83) (Tabla 1).

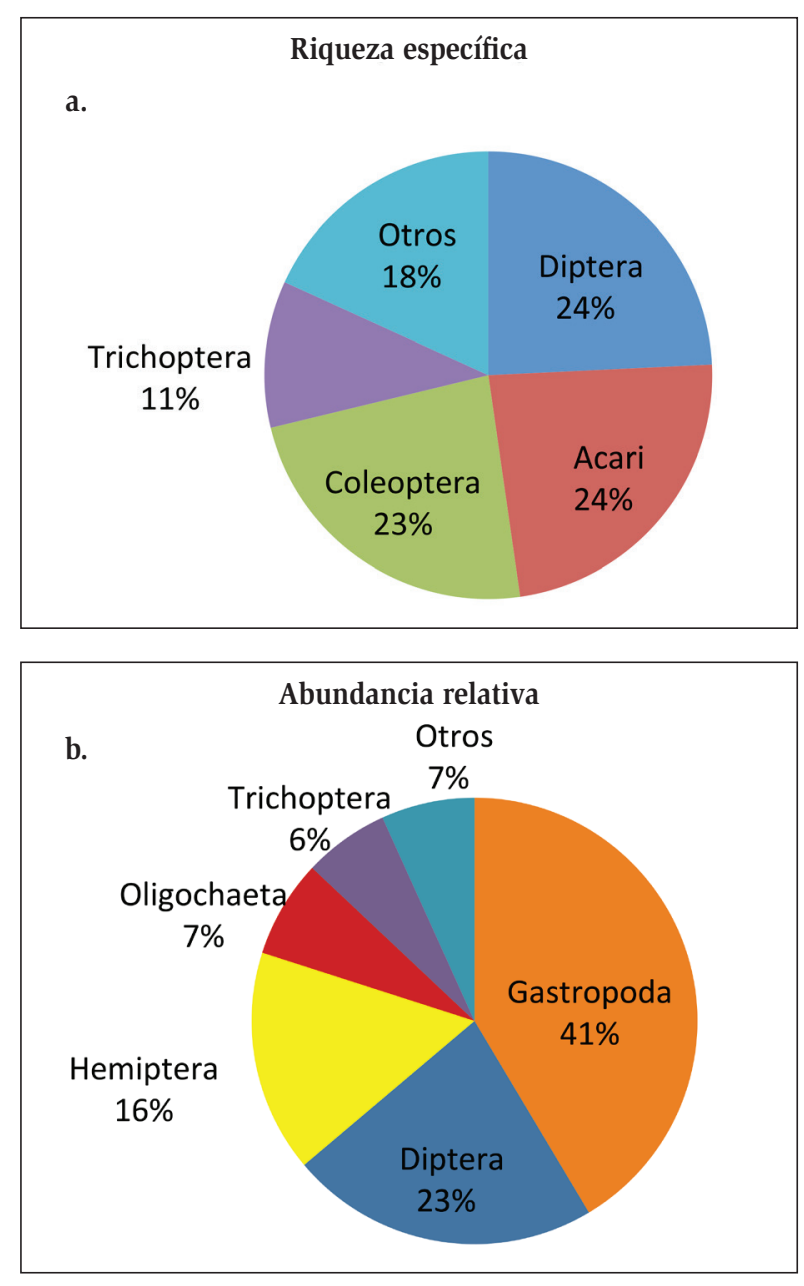

Figura 2a. Porcentaje de riqueza de especies por cada grupo. b. Porcentaje de abundancia de individuos por cada grupo. 
Tabla 1. Descriptores e índices de diversidad para los cinco sitios en cada época de muestreo (1: finalización sequía; 2: lluvias; 3: finalización lluvias) y para el total de cada sitio.

\begin{tabular}{|c|c|c|c|c|c|c|c|c|c|c|c|c|c|c|c|c|c|c|c|c|}
\hline \multirow{2}{*}{$\begin{array}{l}\text { Índice } \\
\text { Época }\end{array}$} & \multicolumn{4}{|c|}{ S Riqueza } & \multicolumn{4}{|c|}{ N Abundancia } & \multicolumn{4}{|c|}{$\begin{array}{c}\text { DMg (Diversidad de } \\
\text { Margalef) }\end{array}$} & \multicolumn{4}{|c|}{$\begin{array}{l}\text { Dominancia de } \\
\text { Simpson (Cinv) }\end{array}$} & \multicolumn{4}{|c|}{$\begin{array}{l}\text { Índice de equidad } \\
\text { Shannon-Wiener }\end{array}$} \\
\hline & 1 & 2 & 3 & Total & 1 & 2 & 3 & Total & 1 & 2 & 3 & Total & 1 & 2 & 3 & Total & 1 & 2 & 3 & Total \\
\hline $\begin{array}{l}\text { Lazy } \\
\text { Hill }\end{array}$ & 26 & 56 & 40 & 75 & 963 & 2022 & 894 & 3879 & 3.6 & 7.2 & 5.7 & 8.9 & 0.2 & 0.2 & 0.1 & 10.8 & 2.0 & 2.5 & 2.7 & 2.8 \\
\hline $\begin{array}{l}\text { Bailey } \\
\text { Spring }\end{array}$ & 25 & 28 & 31 & 57 & 3400 & 2182 & 1578 & 7160 & 3.0 & 3.5 & 4.1 & 6.3 & 0.3 & 0.3 & 0.6 & 3.2 & 1.4 & 1.5 & 1.2 & 1.6 \\
\hline $\begin{array}{l}\text { Fresh } \\
\text { Water }\end{array}$ & 22 & 9 & 11 & 25 & 985 & 235 & 168 & 1388 & 3.0 & 1.5 & 2.0 & 3.3 & 0.3 & 0.2 & 0.4 & 4.2 & 1.6 & 1.8 & 1.5 & 1.9 \\
\hline $\begin{array}{c}\text { El } \\
\text { Pico }\end{array}$ & 31 & 50 & 40 & 77 & 315 & 1588 & 1025 & 2928 & 5.2 & 6.6 & 5.6 & 9.5 & 0.4 & 0.1 & 0.4 & 7.6 & 0.01 & 2.9 & 1.8 & 2.8 \\
\hline $\begin{array}{c}\text { Old } \\
\text { Town } \\
\text { Spring }\end{array}$ & 11 & 30 & 14 & 37 & 174 & 600 & 402 & 1176 & 1.9 & 4.5 & 2.2 & 5.1 & 0.3 & 0.4 & 0.2 & 3.4 & 0.01 & 1.6 & 1.8 & 1.9 \\
\hline Total & 63 & 100 & 72 & 161 & 4967 & 6627 & 4067 & 16531 & 7.3 & 11.3 & 8.5 & 16.5 & 7.6 & 18.0 & 0.1 & 14.2 & 2.7 & 3.6 & 3.2 & 3.5 \\
\hline
\end{tabular}

Cuatro géneros de Chironomidae mostraron una abundancia relativa significativa en los cinco sitios de muestreo y Pyrgophorus platyrachis (Hydrobiidae: Gastropoda) fue de notable abundancia en todos los sitios, excepto en Lazy Hill y en El Pico (Figura 3). En Lazy Hill y en El Pico los dípteros de la familia Chironomidae presentaron las mayores abundancias, mientras que los caracoles de la familia Hydrobiidae mostraron una baja representación en comparación con los otros tres sitios, en donde esta familia tuvo una abundancia mayor que las de las demás familias (Figura 4).

En cuanto a los microhábitats, en general en la hojarasca se encontró la mayor cantidad de individuos (3156), seguido por las raíces (2937 individuos), aunque la mayoría fueron caracoles $P$. platyrachis. En cuanto al número de taxones, en las rocas se obtuvo el mayor número (57), seguido por la hojarasca y los cantos rodados (51 taxones cada uno).

Algunos grupos de especial interés encontrados fueron los ácaros de la familia Halacaridae, efemerópteros del género Hagenulopsis (Leptophlebiidae), y los quironómidos del género Fittkauimyia. La familia Halacaridae se encontró únicamente en Bailey en el

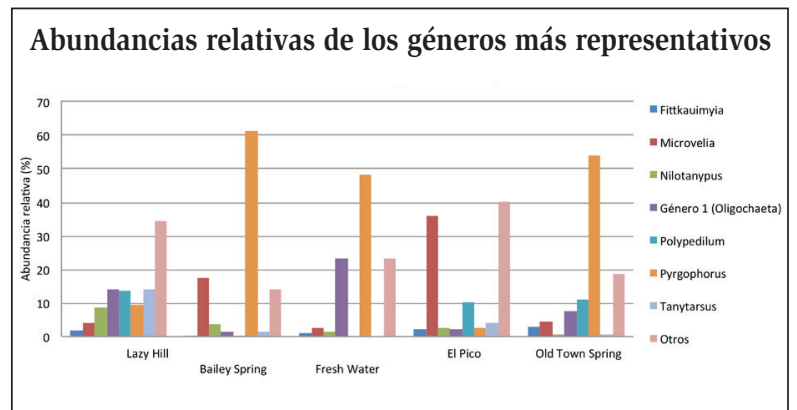

Figura 3. Abundancias relativas de los géneros más representativos en los cinco sitios de muestreo.

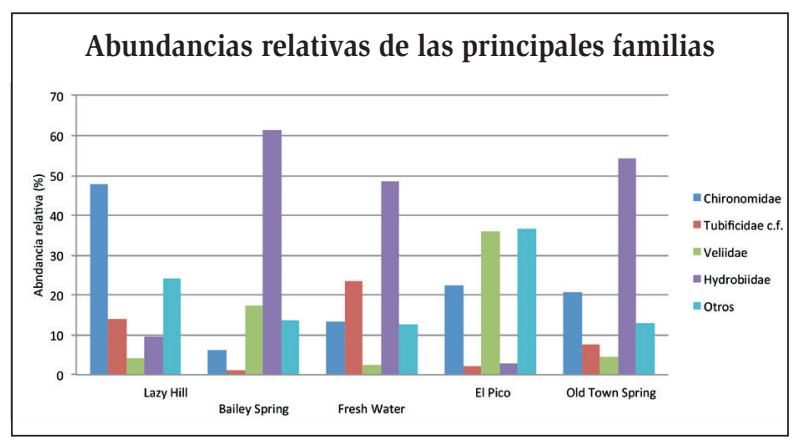

Figura 4. Abundancias relativas de las familias más representativas en los cinco sitios de muestreo. 
primer periodo de muestreo (lluvias bajas), cuando éste presentaba una alta conductividad eléctrica $(1251 \mu \mathrm{S} / \mathrm{cm})$. Los efemerópteros se encontraron en Lazy Hill, El Pico y Old Town, aunque presentaron una baja abundancia (9 individuos). Por último, los quironómidos del género Fittkauimyia se encontraron en los tres periodos de muestreo y en todos los sitios, con una abundancia total de 158 individuos.

\section{Variables físicas y químicas}

En general en la época de lluvias se evidenció un aumento en la concentración de oxígeno disuelto (1.8 $\mathrm{mg} / \mathrm{L}-5.4 \mathrm{mg} / \mathrm{L})$, disminución en la conductividad eléctrica $(1684 \mu \mathrm{S} / \mathrm{cm}-363 \mu \mathrm{S} / \mathrm{cm})$, el pH (7- 6.3), la temperatura $\left(27.9^{\circ} \mathrm{C}-27.4^{\circ} \mathrm{C}\right)$ y la alcalinidad $(3$ $\mathrm{mmol} / \mathrm{L}-0.1 \mathrm{mmol} / \mathrm{L}$ ) (Tabla 2). De acuerdo con el análisis ACC se encontró que en el periodo de lluvias bajas (periodo 1), el eje 1 explica el $55.45 \%$ de la variabilidad, en donde las variables $\mathrm{pH}$ y temperatura fueron las más explicativas (0.629 y -0.627). El eje 2 explica el $39.49 \%$ de la variabilidad y las variables más explicativas allí fueron la conductividad eléctrica $(-0.987)$ y la alcalinidad (-0.852) (Figura 5a.). Esta variabilidad estuvo asociada a los valores más altos de $\mathrm{pH}$, temperatura, conductividad eléctrica y alcalinidad. En el periodo de lluvias, el eje 1 explica el 55.67\% de la variabilidad, y el oxígeno disuelto (0.785) y la conductividad eléctrica $(-0.735)$ fueron las variables más explicativas, mientras que el eje 2 explicó el $33.32 \%$ de la variabilidad, y tuvo asociadas las variables $\mathrm{pH}$ (0.937) y alcalinidad (0.697) (Figura 5b). La variabilidad se asoció a valores altos de oxígeno disuelto, bajos de conductividad y alcalinidad, e intermedios de $\mathrm{pH}$.

En el periodo de finalización de las lluvias, el eje 1 explicó el $53.28 \%$ de la variabilidad, y las variables más explicativas fueron la conductividad eléctrica (0.898) y el pH (-0.773). El eje 2 explicó el $28.42 \%$ de la variabilidad, y la alcalinidad fue la variable más explicativa (-0.974) (Figura 5c). Esto se encuentra relacionado con valores intermedios de conductividad eléctrica y alcalinidad y valores bajos de $\mathrm{pH}$.

Tabla 2. Factores fisicoquímicos medidos en cada época (1: finalización sequía; 2: lluvias; 3: finalización lluvias) en los cinco sitios de muestreo.

\begin{tabular}{cccccccccccccccc}
\hline Sitio & \multicolumn{1}{c}{ Lazy Hill } & \multicolumn{3}{c}{ Bailey } & \multicolumn{4}{c}{ Fresh Water } & \multicolumn{3}{c}{ El Pico } & \multicolumn{5}{c}{ Old Town } \\
\hline Periodo & $\mathbf{1}$ & $\mathbf{2}$ & $\mathbf{3}$ & $\mathbf{1}$ & $\mathbf{2}$ & $\mathbf{3}$ & $\mathbf{1}$ & $\mathbf{2}$ & $\mathbf{3}$ & $\mathbf{1}$ & $\mathbf{2}$ & $\mathbf{3}$ & $\mathbf{1}$ & $\mathbf{2}$ & $\mathbf{3}$ \\
\hline $\begin{array}{c}\text { Oxí́geno } \\
\text { disuelto } \\
(\mathbf{m g} / \mathbf{L})\end{array}$ & 1.8 & 5.4 & 3.6 & 1.8 & 3 & 3.4 & 2.1 & 2 & 1.8 & 2.1 & 3.8 & 4.2 & 2.6 & 4 & 3.6 \\
$\begin{array}{c}\text { Saturación de } \\
\text { Oxígeno \% }\end{array}$ & 21.6 & 67.8 & 42.8 & 23.2 & 37 & 42 & 27.3 & 24.7 & 22.2 & 25.5 & 46.7 & 51.3 & 32.7 & 50.2 & 44.4 \\
$\begin{array}{c}\text { Conductividad } \\
(\boldsymbol{\mu} \text { S/cm) }\end{array}$ & 1684 & 363 & 600 & 1260 & 1234 & 1264 & 630 & 578 & 598 & 425 & 256 & 256 & 950 & 765 & 911 \\
$\begin{array}{c}\text { Temperatura } \\
\left({ }^{\circ} \mathbf{C}\right)\end{array}$ & 26.7 & 26.7 & 24.5 & 26.8 & 26.4 & 25.6 & 27.9 & 27.4 & 26.2 & 26.4 & 26.1 & 24.6 & 26.9 & 27 & 25.8 \\
$\quad \mathbf{p H}$ & 7.5 & 7 & 6.5 & 7.1 & 6.6 & 6.2 & 7.1 & 6.6 & 6.2 & 7.4 & 6.7 & 6.4 & 7 & 6.3 & 6.3 \\
$\begin{array}{c}\text { Alcalinidad } \\
(\mathbf{m m o l} / \mathbf{L})\end{array}$ & 3 & 0.1 & 2 & 2 & 0.3 & 0.3 & 1.2 & 0.3 & 0.5 & 1.6 & 0.1 & 0.3 & 1.5 & 0.3 & 0.8 \\
\hline
\end{tabular}

\section{Variación temporal y espacial}

14 En el periodo 1 (lluvias bajas) se recolectaron 4120 individuos pertenecientes a 51 especies 0 morfoespecies, 46 géneros y 34 familias. En el periodo de lluvias se recolectaron 4873 individuos, 74 especies/ morfoespecies, 63 géneros y 48 familias. En el último periodo (finalización de lluvias) se recolectaron 2644 individuos, 69 especies/morfoespecies, 61 géneros y 45 familias. Adicionalmente, en el periodo de lluvias 
se recolectaron 24 morfotipos que no se encontraron en los otros dos periodos, seis de ellos en Lazy Hill (Acari, 6 morfotipos), seis en El Pico (Tipula, Scirtidae, Hydroscaphidae, Coleoptera, Acari, 2 morfotipos), cuatro en Old Town (Acari, 4 morfotipos), tres en Bailey (Nosodendridae, Acari, Mesovelia) y cinco encontrados en dos de los cinco sitios (Dytiscidae, Berosus, Cyrnellus, Hydrophilidae, 2 morfotipos).

El mayor cambio se dio en Lazy Hill y El Pico (abundancia absoluta, el DMg, y el índice de Simpson), mientras que Bailey mostró poca variación a lo largo de las épocas (DMg y H') (Tabla 1). En general, para la Isla se observó un aumento importante en la diversidad de macroinvertebrados en la época de lluvias (DMg: 9.54) en relación con las otras épocas, a pesar de que en términos de abundancias las diferencias no fueron significativas $(\mathrm{p}>0.05 ; \mathrm{n}=122 ; \mathrm{K}-\mathrm{W}=2.954)$.

En Lazy Hill se recolectó un total de 2514 individuos distribuidos en 57 morfoespecies, 32 de las cuales fueron exclusivas de esta quebrada. La hojarasca fue el microhábitat con mayor número de individuos (1667) y de taxones (23). La prueba de Kruskal Wallis no mostró diferencias significativas entre las abundancias de los tres periodos $(\mathrm{p}>0.05, \mathrm{n}=57, \mathrm{k}-\mathrm{w}=0.5692)$. Además, se encontraron los valores más altos en los índices de Simpson (12.25) y de equidad de Shannon-Wiener (2.89). Después de El Pico, fue el sitio con los valores más altos en riqueza específica (57) y en diversidad (DMg: 7.15).

En Bailey se recolectaron 4241 individuos de 47 morfoespecies. 26 morfoespecies fueron exclusivas para este sitio. La prueba de Kruskal Wallis no mostró diferencias significativas entre las abundancias de los tres periodos $(\mathrm{p}>0.05, \mathrm{n}=47, \mathrm{k}-\mathrm{w}=0.3343)$. En general, el microhábitat de raíces fue el que aportó mayor número de especies (26) e individuos (2937) durante los tres muestreos. Los índices de Simpson y de equidad mostraron los valores más bajos (2.41 y 1.45 , respectivamente), indicando una baja diversidad.

En Fresh Water el microhábitat de hojarasca representó el $68 \%$ de la riqueza y el $71 \%$ de la abundancia. En total se recolectaron 1165 individuos y 21 morfoespecies, ocho de las cuales fueron exclusivas del sitio respecto a los demás. Adicionalmente se encontró el menor valor en
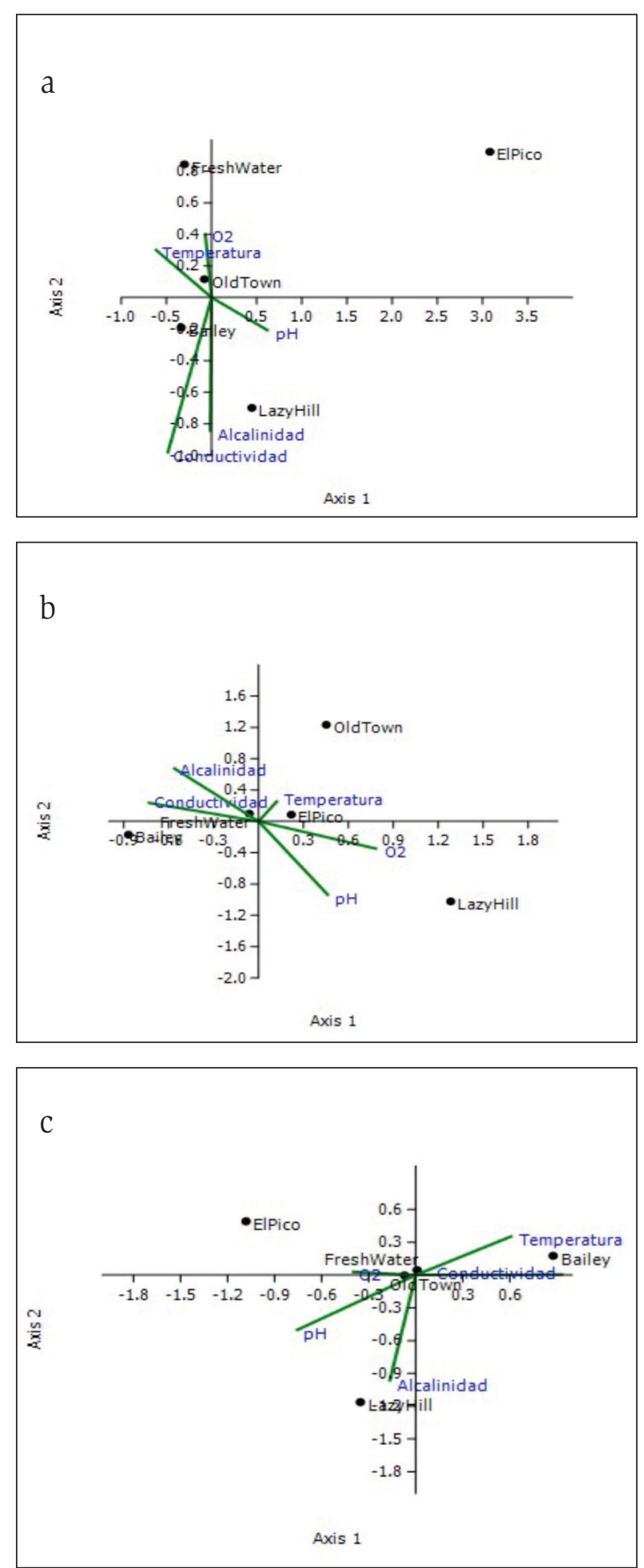

Figura 5. a) ACC para la época 1 (finalización de la sequía). b) ACC para la época 2 (lluvias). c) ACC para la época 3 (finalización de las lluvias). 
el índice de diversidad (DMg: 2.83). No se observaron diferencias significativas en las abundancias entre los muestreos ( $\mathrm{p}>0.05, \mathrm{n}=21, \mathrm{k}-\mathrm{w}=1.793$ ).

En la quebrada El Pico se recolectaron 67 morfoespecies y 2590 individuos, 30 de estas especies fueron exclusivas. La prueba de Kruskal Wallis indicó diferencias significativas en las abundancias entre las épocas de muestreo ( $p<0.05, n=67, k-w=11.6)$. En este sitio se presentaron los valores más altos de riqueza específica (67) y de diversidad (DMg: 8.40). Adicionalmente en la época de sequía, especialmente en el periodo 1 , fue el sitio donde se encontraron el mayor número de especies (27). En la época 1 se registró una gran abundancia de hemípteros patinadores del género Microvelia (Veliidae) que afectó negativamente la diversidad (dominancia de Simpson: 2.31 y H’: 0.01).

Finalmente en Old Town se recolectaron 31 morfoespecies y 1127 individuos, con diez de estas especies siendo exclusivas para el sitio. No se encontraron diferencias significativas en las abundancias entre las épocas de muestreo ( $p>0.05, \mathrm{n}=31, \mathrm{k}-\mathrm{w}=2.016)$. El microhábitat limo fue el que obtuvo el mayor número de taxones (11) y de individuos (960). Este sitio obtuvo la menor abundancia de las quebradas estudiadas.

\section{Distribuciones geográficas}

El $64 \%$ de los géneros encontrados tienen una distribución mundial, presentándose en todas las regiones biogeográficas (con excepción en la Antártida). El 20\% están restringidos a Centro y Suramérica con distribución neotropical y el 16 \% son de distribución americana subtropical.

La revisión de estudios y catálogos de Nicaragua permitió identificar 13 géneros compartidos entre este lugar y la Isla de Providencia (Maes y Mendoza, 1990, Chamorro-Lacayo et al., 2007, Pérez et al., 2008). Los géneros compartidos fueron Leptonema, Smicridea (Hydropsychidae), Cyrnellus (Polycentropodidae), Chimarra (Philopotamidae), Helicopsyche (Helicopsychidae), Neotrichia (Hydroptilidae), Hagenulopsis (Leptophlebiidae), Biomphalaria (Planorbidae), Thysanophora (Thysanophoridae), Neritina (Neritidae), Pyrgophorus (Hydrobiidae), Aedes y Anopheles (Culicidae).

\section{DISCUSIÓN}

En la época de lluvias todos los sitios mostraron un aumento en la diversidad, demostrando así que las comunidades sufren un cambio con la renovación de las aguas debida a las lluvias, aumentando su diversidad, aun en los sitios de carácter predominantemente léntico y a pesar de que los cambios en las abundancias no fueron significativos. Los importantes cambios que demostraron los índices de diversidad para Lazy Hill y para El Pico entre los periodos, permiten inferir que estos son los sitios que están sujetos a la estacionalidad de la Isla, a pesar de la captación de agua en el segundo de ellos. De manera similar, un estudio realizado en una quebrada estacional encontró que los cambios estacionales del caudal favorecen la riqueza y la diversidad de macroinvertebrados, especialmente en la época de lluvias (Longo et al., 2010).

Para el orden Trichoptera se ha registrado una importante riqueza en las Islas del Caribe y Providencia al parecer no es una excepción (12 morfotipos y por lo menos 8 géneros). De acuerdo a lo esperado, este grupo aumentó su riqueza y abundancia en la época de lluvias, además los adultos al no ser buenos voladores, es poco probable que puedan viajar largas distancias. Varias de las especies del Caribe se encuentran restringidas a una o unas pocas islas cercanas entre sí, convirtiéndose las islas en un espacio de evolución y diversificación del grupo (Bass, 2003).

Los Díptera (32 morfoespecies) y los Coleóptera (31 morfoespecies) mostraron ser un elemento importante en la composición de las comunidades de las quebradas de Providencia, donde se evidenció su capacidad de colonización atribuida a la dispersión de los adultos por vuelo activo y por las corrientes de viento (LongoSánchez et al., 2009). Por el contrario, en Providencia los efemerópteros tienen una frecuencia baja, tal como se ha reportado para pequeñas islas neotropicales (Bass, 2003). Los Ephemeróptera pertenecientes al género Hagenulopsis (Leptophlebiidae) han sido reportados para Centro y Suramérica y las Antillas. Grupos como este, que presentan un solo género suelen encontrarse en los ensamblajes de islas, debido a las dificultades inherentes de la colonización (Longo-Sánchez et al., 2009). Los adultos de Ephemeroptera, en particular, 
no vuelan largas distancias y las ninfas no sobreviven al transporte pasivo por medio de otros organismos, debido a sus necesidades de buena condición hídrica, por lo cual es de esperarse que en esta isla de origen volcánico no sea un grupo de particular abundancia.

La familia Chironomidae fue de gran importancia, y además de ser un referente en la abundancia de los sitios, tuvo una riqueza notable. En los sitios de Lazy Hill y El Pico presentó 15 y 14 morfoespecies respectivamente, y diez géneros que pueden encontrarse en ambientes lóticos y lénticos, demostrando la gran capacidad de colonización que tiene el grupo, y el gran aporte en riqueza que hacen a la comunidad en los sitios más diversos de la Isla, como sucede en gran cantidad de ambientes dulceacuícolas sin contaminación orgánica, donde los quironómidos son dominantes (Armitage et al., 1995). Por el contrario, Hydrobiidae estuvo representada solo por $P$. platyrachis y debido a su gran abundancia se encontró dominando los sitios con menor diversidad. Se ha determinado que el género Pyrgophorus tenía su distribución natural restringida a las Islas y al continente alrededor del mar Caribe, y que son especies con potencial invasor en varias partes del mundo y suelen presentarse en altas abundancias (Nava et al., 2011).

En cuanto a los microhábitats, la hojarasca sumergida se resalta en las quebradas de Providencia como el sustrato que soporta las mayores abundancias. Es sabido que en los sistemas lóticos tropicales la hojarasca representa una importante fuente de energía y carbono disponible para el sistema (Dudley-Williams, 2006). Bass (2003) ha sugerido que en los sistemas de quebradas con flujo de agua, la mayoría de la especies prefieren microhábitats de hojarasca, mientras que en los pozos están usualmente asociadas a macrófitas acuáticas.

Otro grupo de especial interés encontrado en la Isla fueron los ácaros de la familia Halacaridae, el cual es predominantemente marino, donde apenas 56 especies han colonizado exitosamente los ambientes dulceacuícolas versus más de 1000 especies marinas (Bartsch, 2008). Aunque tienen una habilidad de dispersión muy baja y baja fecundidad, muchas especies son cosmopolitas, probablemente debido a su carácter generalista y su alta capacidad de tolerancia a numerosos microhábitats, por lo que pueden sobrevivir a corto plazo en condiciones de deterioro ambiental, en aguas subterráneas o superficiales, en manantiales, pozos, zonas hiporréica de los ríos, filtros artificiales, depósitos de arena, entre organismos coloniales, en cámaras branquiales, en musgos y plantas vasculares y en aguas salobres (Bartsch, 2008).

El género Fittkauimyia (Chironomidae: Tanypodinae) ampliamente distribuido en la Isla, presenta larvas que habitan ríos y el litoral de los lagos, generalmente en regiones tropicales y subtropicales y está compuesto por seis especies reportadas para Norteamérica, Japón, Singapur, Indonesia, África, Australia, Centroamérica (Costa Rica) y en la región neotropical sólo en la Amazonía Brasilera (Dantas y Hamada, 2013). Se ha observado que al menos una de sus especies es depredadora "sit-and-wait" (Cranston, 2010; Dantas y Hamada, 2013). En este estudio su presencia es el primer reporte del género para Colombia y para las islas del Caribe, por lo cual se sugieren estudios más detallados con adultos para confirmar la identidad de las especies. Sin embargo, sólo se cuenta con muestras de larvas y exuvias, por lo que un muestreo de adultos sería pertinente para establecer la identidad de las especies.

\section{Variables físicas y químicas}

La disminución de la salinidad y el aumento en la concentración de oxígeno, en el caso de las quebradas de Providencia, obedecerían al aumento del factor de dilución generado por las lluvias, mientras que el agua en movimiento produce oxigenación debido a la turbulencia. Las características cercanas a la neutralidad y la alta conductividad de las aguas en aguas bajas son probablemente generadas por el origen volcánico de la Isla (Blanco, 2009). Por otro lado, las características físicas y químicas de los cuerpos de agua en islas se ve fuertemente influenciada por la conexión con el medio marino y por la misma insularidad (Blanco, 2009), factores que también podrían determinar los altos valores de conductividad presentados. La importancia en particular de la conductividad y la concentración del oxígeno disuelto se reflejó en el Eigenvalue y en los valores de significancia de cada variable presentado en los ACC, especialmente en la época de lluvias. 


\section{Variación temporal y espacial}

Los 24 morfotipos encontrados exclusivamente en el periodo de lluvias confirman nuestra hipótesis, según la cual con las lluvias aparecerían taxones exclusivos y cambiarían las dominancias de éstos.

Lazy Hill es un sitio de resaltar bajo varios aspectos debido a que se encontró la mayor abundancia de crustáceos decápodos que sólo estuvieron presentes en dos quebradas de la isla, a pesar de que suelen ser organismos muy comunes en quebradas tropicales y particularmente en islas debido a su resistencia a la salinidad y la consiguiente capacidad de dispersión (Bass, 2003). Numerosas especies de Atyidae y de Macrobrachium (Palaemonidae) suelen ser comunes en las Islas Caribeñas y se ha reportado que reemplazan a los isópodos y anfípodos de los ecosistemas de regiones templadas, ocupando sus roles funcionales en los trópicos (Bass, 2003). Pyron et al. (1999) encontraron correlaciones significativas en tres arroyos de Puerto Rico entre las abundancias de especies de Atyidae y Macrobrachium con el área, la profundidad y la velocidad del agua. La poca profundidad de la quebrada $(0.15 \mathrm{~m})$ y la velocidad relativamente baja del agua $(0.29 \mathrm{~m} / \mathrm{s})$ podrían explicar la baja abundancia encontrada de estos organismos. Con respecto a los caracoles Neritina punctulata fueron encontrados únicamente en esta quebrada. Covich (1988) registró que numerosas especies de gastrópodos y decápodos tropicales colonizan quebradas dulceacuícolas en el Caribe cerca de la costa marina en las partes bajas, pero son reemplazados por insectos aguas arriba, en tierras más altas, posiblemente por diferencias en las recargas de detritos de hojarasca. Sin embargo, en este caso los organismos fueron encontrados alejados de la costa y a $31 \mathrm{msnm}$, lo cual representa un fenómeno interesante que requiere estudios más detallados. Otros estudios en islas, asocian la presencia de especies de este género a la conectividad permanente o estacional de las quebradas con el mar (Blanco y Scatena, 2006), fenómeno que no se observó en esta quebrada.

El Pico, por su parte, es probablemente la quebrada más significativa de la Isla, en parte por su longitud, ubicada a lo largo de la montaña más alta de Providencia, así como por la importante diversidad que alberga y por el volumen de agua para uso humano que ella aporta. La riqueza específica y el DMg mostraron que éste sitio junto con Lazy Hill, fueron los sitios con la diversidad más alta de la Isla. En particular en la época de lluvias los índices (DMg y H') mostraron que la renovación de las aguas implica una diversificación de la comunidad en la quebrada, en concordancia con lo reportado por Longo et al. (2010). En esta quebrada, al igual que en la quebrada de Fresh Water se observó que en la captación de agua para uso humano no se ha tenido en cuenta el concepto de caudales ecológicos para preservar la funcionalidad del sistema, y aun entrada la época de lluvias se notó que ésta quebrada no tiene un flujo continuo de agua. En ambos sitios, Lazy Hill y El Pico, los importantes valores de riqueza de especies y diversidad de Margalef calculados para el primer periodo permiten suponer que éstos actúan como un refugio para las comunidades durante los meses de estrés hídrico que deben soportar como lo sugiere Dudley-Williams (2006). Los tres sitios restantes presentaron los valores más bajos de diversidad de la Isla, y así mismo son los que presentan las mayores obras de intervención, demostrando la incidencia de éstas en la comunidad de macroinvertebrados. En Bailey los índices de diversidad mostraron una variación mínima, por lo tanto no se puede afirmar que la diversidad del sitio haya mostrado un cambio evidente entre las épocas. Este sitio tuvo el valor de dominancia más alto, los valores más bajos de equidad y la mayor abundancia de individuos, principalmente debido al aporte de $P$. platyrachis, mostrando una distribución desigual para las especies. En Old Town los índices y descriptores fueron particularmente bajos en el primer periodo, $y$ a pesar de que mostraron un aumento con las lluvias, según las pruebas realizadas no hubo diferencias significativas. Al haber sido convertido este sitio en un reservorio permanente de carácter léntico, faltan señales de una sucesión evidente donde las comunidades se estructuran entre un periodo acuático y un periodo seco (Dudley-Williams, 2006).

Fresh Water presentó la menor diversidad de la Isla, aunque las lluvias parecen tener una ligera influencia en la diversidad de la comunidad de macroinvertebrados. Un estudio en la Isla de Guadeloupe (Antillas menores) realizado por Fièvet et al. (2001) estableció los cambios generados en la comunidad de macroinvertebrados (decápodos principalmente) tras la construcción de una represa, entre los que se encuentran la reducción de la distribución de los organismos a lo largo del río, un cambio en la estructura de los gremios, en la 
distribución y diversidad de los taxones y un cambio en la distribución por tallas de los organismos. En las quebradas de Providencia es evidente la falta de un caudal ecológico regulado por la administración de la represa que mantenga un mínimo flujo de agua necesario para el funcionamiento del sistema fluvial.

\section{Distribuciones geográficas}

La mayoría de los macroinvertebrados de las islas del Caribe tienen mecanismos de dispersión que les permiten atravesar aguas oceánicas. Las alas en los adultos o la resistencia a la salinidad como el caso de Neritidae y en algunos crustáceos (Bass, 2003). Islas longevas como Providencia (Plioceno, CORALINA-INVERMAR, 2012), suelen presentar una alta diversidad resultante de la combinación de especies endémicas y especies de amplia distribución (Covich, 2006). En concordancia, los resultados mostraron una diversidad resultante de la combinación de elementos de distribución mundial, neotropical y americana tropical y subtropical, a pesar de que no se encontró ningún género endémico. Bass (2003) resalta que las diferencias entre las clases de macroinvertebrados presentes en Norte y Suramérica y en las Islas del Caribe se presentan a nivel de especies, por lo tanto se requiere un estudio taxonómico más detallado para obtener resultados más concluyentes. Por otro lado, la similitud en la fauna de macroinvertebrados de Nicaragua y los reportados en este estudio (13 géneros compartidos), resalta el importante componente centroamericano de la fauna de Providencia, y también la fuerte influencia que está ejerciendo el continente sobre la Isla, en particular Nicaragua, por ser el país continental más cercano a la Isla.

\section{CONCLUSIONES}

La Isla de Providencia contiene una fauna de macroinvertebrados acuáticos diversa, producto de la convergencia de elementos de distribución mundial, elementos subtropicales y elementos tropicales típicamente centro y suramericanos.

Se comprobó que la renovación de las aguas generada durante el periodo de lluvias, favorece la diversidad de las comunidades de macroinvertebrados acuáticos en las quebradas de la isla, con un aumento en su composición, pero no en su abundancia. Adicionalmente durante el periodo de lluvias, aparecen taxones que no se encontraron durante los periodos más secos, y cambia la dominancia de los taxones.

Los patrones de variación de las comunidades encontrados en este trabajo indican que las quebradas de El Pico y Lazy Hill son las que soportan una mayor diversidad faunística a lo largo del año. Esto debido a que aún conservan la estructura del cauce, a diferencia de los tres sitios restantes, en donde las estructuras de captación han transformado los sistemas lóticos en unos predominantemente lénticos.

\section{AGRADECIMIENTOS}

A la Corporación Coralina por permitirnos el trabajo de campo en la Isla. A la División de Investigación DIB Bogotá de la Universidad Nacional de Colombia por su apoyo financiero para el desarrollo del proyecto. Al Profesor Edgar Linares por su ayuda en la identificación del material biológico.

\section{BIBLIOGRAFÍA}

Alarie, Y., M.C. Michat, A.N. Nilsson, M. Archangelsky y L. Hendrich. 2009. Larval morphology of Rhantus Dejean, 1833 (Coleoptera: Dytiscidae: Colymbetinae): descriptions of 22 species and phylogenetic considerations. Zootaxa 2317: 1- 102.

Armitage, P.D., P.S. Cranston y L.C.V. Pinder. 1995. The Chironomidae Biology and ecology of non-biting midges. Chapman \& Hall First edition. 572 p.

Ashe, P., D.A. Murray y F. Reiss. 1987. The zoogeographical distribution of Chironomidae (Insecta: Diptera). Annales de Limnologie 23 (1): 27- 60.

Bartsch, I. 2008. Global diversity of halacarid mites (Halacaridae: Acari: Arachnida) in freshwater. Hydrobiologia 595: 317-322.

Bass, D. 2003. A comparison of freshwater macroinvertebrate communities on small Caribbean Islands. BioScience 53 (11): 1094-1100.

Blahnik, R.J. y R.W. Holzenthal. 2012. New neotropical species of Chimarra (Trichoptera, Philopotamidae). ZooKeys 184: 1- 33.

Blanco, J.F. 2009. Características físico-químicas de las quebradas del Parque Nacional Natural Gorgona, Pacífico Colombiano. Actualidades Biológicas 31 (91): 123-140. 
Blanco, J.F. y F.N. Scatena. 2006. Hierarchical contribution of river-ocean connectivity, water chemistry, hydraulics, and substrate to the distribution of diadromous snails in Puerto Rican streams. Journal of the North American Benthological Society 25 (1): 82-98.

Borkent, A. y G.R. Spinelli. 2007. Neotropical Ceratopogonidae (Diptera: Insecta). 199 pp. En: Adis J, Arias JR, RuedaDelgado G, Wantzen K. M (Ed). Biodiversidad Acuática en América Latina. Pensoft, Sofia, Moscow. 685 p.

Cartwright, D.I. 2009. Austrotinodes Schmid, a South and Central American caddisfly genus, newly recorded in Australia, with the description of new species (Trichoptera: Ecnomidae). Zootaxa 2142: 1- 19.

Chamorro-Lacayo, M. L., J. M. Maes, R. J. Blahnik y R. W. Holzenthal. 2007. Checklist of the Trichoptera of Nicaragua. pp. 37-50. En: Bueno-Soria J., R. Barba-Alvarez, B. Armitage (Ed). Proceedings of the 12th International Symposium on Trichoptera. The Caddis Press, Ciudad de México, 378 p.

CORALINA- INVEMAR. 2012. Gómez-López D.I, C. SeguraQuintero, P.C Sierra-Correa y J Garay-Tinoco (Ed). Atlas de la Reserva de la Biósfera Seaflower. Archipiélago de San Andrés, Providencia y Santa Catalina. Instituto de investigaciones Marinas y Costeras "José Benito Vives de Andréis” -INVEMAR- y Corporación para el Desarrollo sostenible del Archipiélago de San Andrés, Providencia y Santa Catalina -CORALINA-. Serie de publicaciones Especiales de INVEMAR \#28. Santa Marta, Colombia 180 p.

Covich, A.P. 1988. Geographical and historical comparisons of neotropical streams: Biotic diversity and detrital processing in highly variable habitats. Journal of the North American Benthological Society 7: 361-368.

Covich, A.P. 2006. Dispersal-limited biodiversity of tropical insular streams. Polish Journal of Ecology 54 (4): 523-547.

Cranston, P. 2010. Chiro Key. Documento web URL: http:// chirokey.skullisland.info/. Consultado: 15 enero 2014.

Dantas, G.P.S. y N. Hamada. 2013. Two new species of Fittkauimyia Karunakaran (Diptera: Chironomidae) from Brazil. Zootaxa 3681 (5): 573-582.

DeJong, R.J., J.A.T. Morgan, W. L. Paraense, J.P. Pointier, M. Amarista, P.F.K. Ayeh-Kumi, A. Babiker, C. S. Barbosa, P. Brémond, A. P. Canese, C. Pereira de Sousa, C. Dominguez, S. File, A. Gutierrez, R. N. Incani, T. Kawano, F. Kazibwe, J. Kpikpi, N. J. S. Lwambo, R. Mimpfoundi,
F. Njiokou, J. N. Poda, M. Sene, L. E. Velásquez, M. Yong, C. M. Adema, B. V. Hofkin, G. M. Mkoji, y E. S. Loker. 2001. Evolutionary relationships and biogeography of Biomphalaria (Gastropoda: Planorbidae) with implications regarding its role as host of the human bloodfluke, Schistosoma mansoni. Molecular biology and evolution 18 (12): 2225- 2239.

Deler-Hernández, A., M. Fikáček y F. Cala-Riquelme. 2013. A review of the genus Berosus Leach of Cuba (Coleoptera, Hydrophilidae). ZooKeys 273: 73- 106.

Díaz-Merlano, J.M. y M. Puyana-Hegedus. 1994. Moluscos del Caribe colombiano. Un catálogo ilustrado. Colciencias, Fundación Natura, INVEMAR. 291 p.

Domínguez, E. y H.R. Fernández (Ed). 2009. Macroinvertebrados bentónicos suramericanos. Sistemática y biología. Fundación Miguel Lillo, Tucumán. 211 p.

Dudley-Williams, D. 2006. The Biology of Temporary Waters. Oxford University Press. 348 p.

Epler, J.H. 1992. Identification manual for the Larval Chironomidae (Diptera) of Florida. Department of Environmental Regulation. State of Florida. 1995 p.

Fièvet, E, S. Dolédec y P. Lim. 2001. Distribution of migratory fishes and shrimps along multivariate gradients in tropical island streams. Journal of Fish Biology 59: 390-402.

García, H.A. 2002. Los hemípteros de la película superficial del agua en Colombia. Parte I. Familia Gerridae. Academia colombiana de Ciencias exactas, físicas y naturales. Colección Jorge Álvarez Lleras No. 20: 239.

Hájek, J. y J. Ŝtastnŷ. 2005. Two new species of Laccophilus Leach, 1815 (Coleoptera: Dytiscidae) from India and Borneo with notes on other species of the genus. Acta entomologica Musei Nationalis Pragae 45: 51- 58.

Harris, S.C. 1990. New species of Neotrichia from Central and South America. Journal of the New York Entomological Society 98 (2): 246- 260.

Heckman, C.W. 2001. Encyclopedia of South American Aquatic Insects: Collembola. Springer Science Business Media Dordrecht, $414 \mathrm{p}$.

Heckman, C.W. 2006. Encyclopedia of South American Aquatic Insects: Odonata-Anisoptera. Netherlands: Springer, $730 \mathrm{p}$. 
Longo-Sánchez, M.C., A.M. Gómez-Aguirre, J.F. Blanco y H. Zamora-González. 2009. Cambios multianuales y espaciales de la composición y estructura del ensamblaje de insectos acuáticos en las quebradas perennes de la Isla Gorgona, Colombia. Actualidades Biológicas 31 (91): 141-160.

Longo, M., H. Zamora, C. Guisande, J.J. Ramírez. 2010. Dinámica de la comunidad de macroinvertebrados en la quebrada Potrerillos (Colombia): Respuesta a los cambios estacionales de caudal. Limnetica 29 (2): 195-210.

Maes, J.M. y P.R. Mendoza. 1990. Catálogo de los Díptera de Nicaragua. Revista nicaragüense de entomología 14: 19-39.

Michat, M.C. y P.L.M. Torres. 2006. Hydaticus tuyuensis Trémouilles (Coleoptera: Dytiscidae): larval morphology and phylogenetic relationships within Dytiscinae. Hydrobiologia 563: 479- 492.

Muñoz-Riveaux, S., C. Naranjo-López, G. GarcésGonzález, D.D. González Lazo, Y. Musle-Cordero y L. Rodríguez-Montoya. 2003. Evaluación de la calidad del agua utilizando los macroinvertebrados bentónicos como bioindicadores. Revista Chapingo 9 (2): 147-153.

Naranjo-López, C., D.D. González-Lazo, G. GarcésGonzález, A.L. Brandimarte, S. Muñoz-Riveaux y Y. Musle-Cordero. 2005. Una metodología rápida y de fácil aplicación para la evaluación de la calidad del agua utilizando el índice BMWP-Cub para ríos cubanos. Tecnura 9 (17): 65-76.

Nava, M., H. Severeyn y N. Machado. 2011. Distribución y taxonomía de Pyrgophorus platyrachis (Caenogastropoda: Hydrobiidae), en el Sistema de Maracaibo, Venezuela. Revista de Biología Tropical, 59 (3): 1165-1172.

Nessimian, J.L. y L.L. Dumas. 2010. Description of the immature stages of Leptonema tridens (Insecta: Trichoptera: Hydropsychidae) from southeastern Brazil with notes on its biology. Zoologia 27 (3): 465- 471.

Oyewo, E.A. y O.A. Sæther. 2008. Revision of Polypedilum (Pentapedilum) Kieffer and Ainuyusurika Sasa et Shirasaki (Diptera: Chironomidae). Zootaxa 1953: 1-145.

Palacino-Rodríguez, F. 2011. Taxonomía y filogenia del género Erythemis Hagen, 1861 (Odonata: Libellulidae). Tesis de maestría, Universidad Nacional de Colombia, Bogotá, Colombia. 141 pp.
PAST Paleontological Statistics. 1999-2013. [programa de ordenador] Version 3.0. University of Oslo.

Pérez, A.M., M. Sotelo, I. Arana y A. López. 2008. Diversidad de moluscos gasterópodos terrestres en la región del Pacífico de Nicaragua y sus preferencias de hábitat. Revista de Biología Tropical 56 (1): 317-332.

Pérez-Pompa, N.E., A.M. Marañón-Reyes, A. GonzálezMarañón, Y. Rodríguez-Mendoza y C. Naranjo-López. 2012. Estudio de la correlación entre el índice biótico BMWP-Cub y parámetros físico-químicos en el río Gascón de Santiago de Cuba. Revista Cubana de Química 24 (3): 231-242.

Pyron, M., A.P. Covich y R.W. Black. 1999. On the relative importance of pool morphology and woody debris to distributions of shrimps in a Puerto Rican headwater stream. Hydrobiologia 405: 207-215.

Riss, W., R. Ospina y J.D. Gutiérrez. 2002. Establecimiento de los valores de bioindicación para macroinvertebrados acuáticos de la sabana de Bogotá. Caldasia 24 (1): 135-156.

Ruiz-Moreno, J.L., R. Ospina-Torres y W. Riss. 2000. Guía para la identificación genérica de larvas de quironómidos (Diptera: Chironomidae) de la Sabana de Bogotá. II. Subfamilia Chironominae. Caldasia 22 (1): 15-33.

Sganga, J.V. y E.B. Angrisano. 2005. El género Smicridea (Trichoptera: Hydropsychidae: Smicrideinae) en el Uruguay. Revista de la sociedad entomológica argentina 64 (3): 131-139.

Smit, H. 2004. The water mite genus Koenikea Wolcott from Australia (Acari: Hydrachnidia: Unionicolidae). Records of the Western Australian Museum 22: 165- 191.

Taylor, D.W. 2003. Introduction to Physidae (Gastropoda: Hygrophila); biogeography, classification, morphology. Revista de Biología Tropical. 51 (Suppl. 1): 1- 287.

Toledo, M., Y.S. Megna y Y. Alarie. 2011. Description of a new species of Laccodytes Régimbart, 1895 (Coleoptera, Dytiscidae, Laccophilinae) from Cuba. Zootaxa 2792: 63- 67.

Triplehorn, C.A. y N.F. Johnson (Ed). 2005. Borror and Delong's Introduction to the Study of Insects. United States of America: Thomson Brooks/Cole. 864 p.

Valencia, D. y M. Campos. 2007. Freshwater prawns of the genus Macrobrachium Bate, 1868 (Crustacea: Decapoda: Palaemonidae) of Colombia. Zootaxa 1456: 1-44. 
Wiederholm, T. (Ed). 1983. Chironomidae of the Holartic region. Keys and diagnoses. Part 1- Larvae. Borgströms Trykeri AB. Motala. 573 p.

Wiederholm, T. (Ed). 1986. Chironomidae of the Holartic region. Keys and diagnoses. Part 2- Pupae. Entomologica Scandinavica Supplement No 28. Lund. 1471 p.
Woolley, T.A. 1969. Two new species of Hydrozetes, Extant and fossil (Acari: Cryptostigmata, Hydrozetidae). Journal of the New York Entomological Society 77 (4): 250- 256.

Fecha de Recepción: 26/05/2014 Fecha de Aceptación: 13/11/2014

Para citar este artículo: Cortés-Guzmán, D y R. Ospina-Torres. 2014. Comunidad de Macroinvertebrados acuáticos en quebradas de la Isla de Providencia, Mar Caribe Colombiano. Revista Intrópica 9: 9 - 22 